\title{
Polynomials Orthogonal on the Semicircle
}

\author{
Lothar Reichel*
}

\section{References}

[WG1] W. Gautschi and G. V. Milovanović, Polynomials orthogonal on the semicircle, J. Approx. Theory, 46 (1986), pp. 230-250.

[WG2] W. Gautschi, H. J. Landau, and G. V. Milovanović, Polynomials orthogonal on the semicircle, II, Constr. Approx., 3 (1987), pp. 389-404.

\section{Introduction}

In the above two papers Walter Gautschi, jointly with Henry J. Landau and Gradimir V. Milovanović, investigate polynomials that are orthogonal with respect to a non-Hermitian inner product defined on the upper half of the unit circle in the complex plane. For special choices of the weight function, these polynomials are related to Jacobi polynomials. Their recurrence relation and properties of their zeros are investigated, and applications to Gauss quadrature are explored. We first discuss the importance of orthogonal polynomials that satisfy recurrence relations with few terms, and then focus on the special properties of orthogonal polynomials on the semicircle.

\section{Recurrence relations for orthogonal polyno- mials}

Orthogonal polynomials are important in analysis, approximation theory, and computational mathematics. They provide a convenient basis both to express and compute polynomial approximants. The $n \times n$ matrix determined by the recursion coefficients for the first $n+1$ orthogonal polynomials is helpful for computing the nodes and weights of the $n$-point Gauss quadrature rule. Moreover, orthogonal polynomials form the foundation for numerous iterative methods in linear algebra, including the conjugate gradient method for the solution of large linear systems of equations with a symmetric positive definite matrix, and the

\footnotetext{
${ }^{*}$ Department of Mathematical Sciences, Kent State University, Kent, OH 44242, USA. Research supported in part by NSF grant DMS-1115385. E-Mail: reichel@math.kent.edu.
} 
symmetric Lanczos process for the computation of a few selected eigenvalues and associated eigenvectors of a large symmetric matrix. Very nice discussions of the many applications of orthogonal polynomials in scientific computation is provided by Gautschi in his survey article [8] as well as in his wonderful book [9]. Further examples of usage of orthogonal polynomials in linear algebra can be found in the recent book by Golub and Meurant [11].

The symmetric Lanczos process is a manifestation of the Stieltjes procedure for generating the recursion coefficients for polynomials orthogonal with respect to an inner product on a real interval. These polynomials satisfy a three-term recurrence relation. The symmetric Lanczos process is the foundation for the conjugate gradient method for the iterative solution of large linear systems of equations with a symmetric positive definite matrix. The existence of a recurrence relation for the orthogonal polynomials with few terms reduces the computational effort and storage requirement for the conjugate gradient method. The availability of these recurrence relations also reduces the computational effort required by the symmetric Lanczos process for the computation of a few selected eigenvalues and associated eigenvectors of a large symmetric matrix. Analogously, the three-term recurrence relation for orthogonal polynomials is fundamental for the efficiency of the QR algorithm for the computation of all eigenvalues and possibly eigenvectors of a symmetric matrix. A variant of this algorithm is commonly applied to compute the nodes and weights of Gauss quadrature rules associated with a positive measure on a real interval; see, e.g., $[8,11]$. The use of orthogonal polynomials for data-fitting is natural and the existence of a three-term recursion relation reduces the computational effort; see, e.g., Elhay et al. [6] and Gautschi [8].

The above discussion illustrates that the existence of orthogonal polynomials that satisfy a recurrence formula with few terms is of significant interest in scientific computation. The aforementioned numerical methods use the threeterm recurrence relations of polynomials that are orthogonal with respect to an inner product defined by a nonnegative measure on a real interval. Also matrixvalued polynomials that are orthogonal with respect to a symmetric positive definite matrix-valued measure satisfy a three-term recurrence relation and find applications in scientific computation; see, e.g., $[2,11]$.

Polynomials that satisfy short recurrence relations different from three-term relations are of interest in computations as well. For instance, Szegő polynomials and the associated reversed polynomials satisfy pairs of short recurrence relations. Szegó polynomials find numerous applications in statistics and signal processing $[14,16]$, and their recurrence relations form the basis for an efficient QR algorithm for computing all eigenvalues, and possibly also all eigenvectors, of a unitary upper Hessenberg matrix [12,24]. This QR algorithm can be applied to compute the nodes and weights of Gauss-Szegö quadrature rules associated with a positive measure on the unit circle $[13,15]$. The short recurrence relations of Szegö polynomials are important for the development of efficient algorithms for data-fitting applications as well; see, e.g., [1].

Orthogonal polynomials with respect to most inner products with support in the complex plane do not satisfy a short recurrence relation or pairs of short 
recurrence relations. For this reason the inner product sometimes is replaced by a bilinear form chosen to obtain families of polynomials that satisfy short recurrence relations. These polynomials give rise to oblique projection methods, such as the nonsymmetric Lanczos process, and they can be used in iterative methods for the solution of large linear systems of equations with a square nonsymmetric matrix, and for the computation of a few eigenvalues and associated eigenvectors of such a matrix; see, e.g., Brezinski [3] for discussions and applications. A difficulty with these methods is that the recurrence formulas may break down and then require a special recovery procedure; see, e.g., Brezinski et al. [4] and references therein.

In 1985, before the publication of the first paper by Gautschi and Milovanović [10] on orthogonal polynomials on the semicircle, only polynomials orthogonal with respect to an inner product on an interval or on a circle were known to satisfy recurrence relations with few terms and not to suffer from the possibility of break down. The results of this paper and of the more complete investigations [WG1, WG2] therefore were quite surprising. The uncovering of the many nice properties of orthogonal polynomials on the semicircle was very important for analysis, approximation theory, and computational mathematics, and has spurred related work. The following section describes some important properties of orthogonal polynomials on the semicircle and the last section discusses some applications and more recent work.

\section{Orthogonal polynomials on the semicircle}

Let $w$ be a weight function that is positive at infinitely many points in the open interval $(-1,1)$, is integrable on this interval, and can be extended to a function $w(z)$ holomorphic in the open unit half disc $D_{+}=\{z \in \mathbb{C}:|z|<1, \operatorname{Im}(z)>0\}$; the function $w$ may be singular at \pm 1 . Introduce the inner product

$$
(f, g)=\int_{\Gamma} f(z) g(z) w(z)(i z)^{-1} d z=\int_{0}^{\pi} f\left(e^{i \theta}\right) g\left(e^{i \theta}\right) w\left(e^{i \theta}\right) d \theta
$$

where $i=\sqrt{-1}$ and $\Gamma$ is the upper unit semicircle in the complex plane $\mathbb{C}$. Also define the inner product

$$
[f, g]=\int_{-1}^{1} f(x) \bar{g}(x) w(x) d x,
$$

where the bar denotes complex conjugation. All integrals are assumed to exist, possibly as suitably defined improper integrals. The inner product (2) is positive definite. Therefore, there is a family of infinitely many monic orthogonal polynomials $\left\{p_{j}\right\}_{j=0}^{\infty}$ such that

$$
\left[p_{j}, p_{k}\right]\left\{\begin{array}{ll}
>0, & j=k, \\
=0, & j \neq k,
\end{array} \quad j, k=0,1,2, \ldots .\right.
$$


The inner product (1) is non-Hermitian. It therefore is not obvious that there is a family of infinitely many monic orthogonal polynomials $\left\{\pi_{j}\right\}_{j=0}^{\infty}$ such that

$$
\left(\pi_{j}, \pi_{k}\right)\left\{\begin{array}{ll}
\neq 0, & j=k, \\
=0, & j \neq k,
\end{array} \quad j, k=0,1,2, \ldots .\right.
$$

Gautschi et al. [WG2] showed that under the mild restriction

$$
\operatorname{Re}(1,1)=\operatorname{Re}\left(\int_{0}^{\pi} w\left(e^{i \theta}\right) d \theta\right) \neq 0
$$

the orthogonal polynomials $\pi_{j}$ exists. Moreover, they can be expressed in terms of the orthogonal polynomials $p_{j}$. Specifically, we have

$$
\pi_{k}(z)=p_{k}(z)-i \theta_{k-1} p_{k-1}(z), \quad k=0,1,2, \ldots, \quad p_{-1}(z)=0,
$$

where

$$
\theta_{k-1}=\frac{\mu_{0} p_{k}(0)+i q_{k}(0)}{i \mu_{0} p_{k-1}(0)-q_{k-1}(0)}, \quad k=0,1,2, \ldots .
$$

The $q_{k}(0)$ denote the values of the associated polynomials

$$
q_{k}(z)=\int_{-1}^{1} \frac{p_{k}(z)-p_{k}(x)}{z-x} w(x) d x, \quad q_{-1}(z)=-1
$$

at the origin, and $\mu_{0}=(1,1)$ is the zeroth moment with respect to the inner product (1). Using the fact that the polynomials $p_{k}$ satisfy a three-term recurrence relation, Gautschi et al. [WG2] obtain from (3) that the polynomials $\pi_{k}$ satisfy a three-term recurrence relation of the form

$$
\pi_{k+1}(z)=\left(z-i \alpha_{k}\right) \pi_{k}(z)-\beta_{k} \pi_{k-1}(z), \quad k=0,1,2, \ldots
$$

with $\pi_{-1}(z)=0$ and $\pi_{0}(z)=1$.

The recursion formula (4) indicates that the eigenvalues of the tridiagonal matrix

$$
J_{k}=\left[\begin{array}{cccccc}
i \alpha_{0} & 1 & & & & 0 \\
\beta_{1} & i \alpha_{1} & 1 & & & \\
& \beta_{2} & i \alpha_{2} & 1 & & \\
& & \ddots & \ddots & \ddots & \\
0 & & & & \beta_{k-1} & i \alpha_{k-1}
\end{array}\right] \in \mathbb{C}^{k \times k}
$$

are the zeros of $\pi_{k}$.

If the weight function $w$ satisfies $w(z)=w(-z)$ and $w(0)>0$, then the subdiagonal entries of the matrix $J_{k}$ are real, and $J_{k}$ can be transformed to a real matrix. Moreover, Gautschi et al. [WG2, Theorem 6.2] show that all zeros of the orthogonal polynomials $\pi_{k}$ live in the open upper half of the unit disk in $\mathbb{C}$ except possibly for a single zero on the positive imaginary axis. 
Finally, Gautschi et al. [WG2, Theorem 6.2] discuss the special case of Jacobi- and Gegenbauer-type weight functions. The latter are given by

$$
w(z)=\left(1-z^{2}\right)^{\lambda-1 / 2}, \quad \lambda>-\frac{1}{2},
$$

where the fractional powers are understood in terms of their principal branches. The zeros of the orthogonal polynomials $\pi_{k}, k \geq 2$, associated with a Gegenbauertype weight function (5) are shown to be simple, distributed symmetrically with respect to the imaginary axis, and contained in the open upper unit half disc. Further results on the zeros of polynomials orthogonal on the semicircle are presented by Gautschi in [7].

\section{Extensions and applications}

A fairly natural modification of the work by Gautschi et al. [WG1, WG2] is to consider an inner product on a subarc of the upper half of the unit circle. de Bruin [5] investigated properties of polynomials orthogonal with respect to a possibly non-Hermitian inner product on an arc of the unit circle, symmetric with respect to the imaginary axis. Functions of the second kind and Stieltjes polynomials for such inner products are described by Milovanović and Rajković [22]. Milovanović [19] discusses Gauss quadrature rules and provides error bounds for integrals defined on the semicircle. Relations of polynomials orthogonal on the semicircle or on a circular arc to polynomials orthogonal with respect to an inner product on certain contours in $\mathbb{C}$ are explored by Milovanović and Rajković [21]. Applications of orthogonal polynomials on the semicircle to differentiation is described by Caliò et al. [20], and their use in zero-finders are commented on by Petković et al. [23]; see also Milovanović $[17,18]$ for discussions on applications. A recent account of the orthogonal polynomials on the semicircle can be found in Gautschi [9, Section 1.8].

Acknowledgement. I would like to thank Gradimir Milovanović for comments and references.

\section{References}

[1] G. S. Ammar, W. B. Gragg, and L. Reichel, Downdating of Szegö polynomials and data fitting applications, Linear Algebra Appl., 172 (1992), pp. $315-336$.

[2] J. Baglama, D. Calvetti, and L. Reichel, IRBL: An implicitly restarted block Lanczos method for large-scale Hermitian eigenproblems, SIAM J. Sci. Comput., 24 (2003), pp. 1650-1677.

[3] C. Brezinski, Biorthogonality and its Applications to Numerical Analysis, Marcel Dekker, New York, 1992. 
[4] C. Brezinski, M. Redivo Zaglia, and H. Sadok. New look-ahead Lanczos-type algorithms for linear systems, Numer. Math., 83 (1999), pp. 53-85.

[5] M. G. de Bruin, Polynomials orthogonal on a circular arc, J. Comput. Appl. Math., 31 (1990), pp. 253-266.

[6] S. Elhay, G. H. Golub, and J. Kautsky, Updating and downdating of orthogonal polynomials with data-fitting applications, SIAM J. Matrix Anal. Appl., 12 (1991), pp. 327-353.

[7] W. Gautschi, On the zeros of polynomials orthogonal on the semicircle, SIAM J. Math. Anal., 20 (1989), pp. 738-743.

[8] W. Gautschi, The interplay between classical analysis and (numerical) linear algebra - a tribute to Gene H. Golub, Electron. Trans. Numer. Anal., 13 (2002), pp. 119-147.

[9] W. Gautschi, Orthogonal Polynomials: Computation and Approximation, Oxford University Press, Oxford, 2004.

[10] W. Gautschi and G. V. Milovanović, Polynomials orthogonal on the semicircle, Rend. Sem. Mat. Univ. Politec. Torino, 1985, special issue, pp. 179185.

[11] G. H. Golub and G. Meurant, Matrices, Moments and Quadrature with Applications, Princeton University Press, Princeton, 2009.

[12] W. B. Gragg, The QR algorithm for unitary Hessenberg matrices, J. Comput. Appl. Math., 16 (1986), pp. 1-8.

[13] W. B. Gragg, Positive definite Toeplitz matrices, the Arnoldi process for isometric operators, and Gaussian quadrature on the unit circle, J. Comput. Appl. Math., 46 (1993), pp. 183-198.

[14] U. Grenander and G. Szego,, Toeplitz Forms and Applications, Chelsea, New York, 1984.

[15] W. B. Jones, O. Njåstad, and W. J. Thron, Moment theory, orthogonal polynomials, quadrature and continued fractions associated with the unit circle, Bull. London Math. Soc., 21 (1989), pp. 113-152.

[16] T. Kailath, Linear estimation for stationary and near-stationary processes, in T. Kailath, ed., Modern Signal Processing, Hemisphere Publ., Washington, DC, 1985, pp. 59-128.

[17] G. V. Milovanović, Some applications of the polynomials orthogonal on the semicircle, in Numerical Methods (Miskolc, 1986), Colloquia Mathematica Societatis Janos Bolyai, Vol. 50, North-Holland, Amsterdam, 1987, pp. 625-634. 
[18] G. V. Milovanović, Complex orthogonality on the semicircle with respect to Gegenbauer weight: Theory and applications, in Topics in Mathematical Analysis, ed. Th. M. Rassias, World Scientific Publ., Teaneck, NJ, 1989, pp. 695-722.

[19] G. V. Milovanović, On polynomials orthogonal on the semicircle and applications, J. Comput. Appl. Math., 49 (1993), pp. 193-199.

[20] F. Calio', M. Frontini, G.V. Milovanović, Numerical differentiation of analytic functions using quadratures on the semicircle, Comput. Math. Appl., 22 (1991), pp. 99-106.

[21] G. V. Milovanović and P. M. Rajković, Geronimus concept of orthogonality for polynomials orthogonal on a circular arc, Rend. Mat Appl. (7), 10 (1990), pp. 383-390.

[22] G. V. Milovanović and P. M. Rajković, On polynomials orthogonal on a circular arc, J. Comput. Appl. Math., 51 (1994), pp. 1-13.

[23] M. S. Petkovic, T. Sakurai, and L. Rančić, Family of simultaneous methods of Hansen-Patrik's type, Appl. Numer. Math., 50 (2004), pp. 489-510.

[24] M. Stewart, An error analysis of a unitary Hessenberg QR algorithm, SIAM J. Matrix. Anal. Appl., 28 (2006), pp. 40-67. 